\title{
Sexual behaviours of HIV positive adults receiving HAART in Botswana: a cross sectional study
}

\author{
Mpho Keetile, Gofaone Kgosidintsi
}

University of Botswana, Department of Population Studies, Private Bag 00705,Gaborone

\begin{abstract}
Objective: This paper aims to assess the sexual behaviour of HIV positive adult men and women on HAART in Botswana. Methods: Data derived from the Botswana AIDS Impact Survey IV (BAIS IV-2013) was used. A sample of 1065 HIV positive men and women in age range of 20-49 years were considered for analysis.

Results: From the sample of a total of 1065 HIV positive adults only $22 \%$ (238) were on HAART. About 2\% of respondents in the total sample were involved in transactional sex, $9 \%$ reported that they had multiple sexual partners in the past 12 months, $6 \%$ reported that they were once involved in inter-generational sexual relationships. Meanwhile, for respondents on HAART, $8 \%$ reported multiple sexual partners in the past 12 months, while $5 \%$ had been involved in inter-generational sex, about $2 \%$ had been involved in transactional sex and 13\% had not used condoms consistently. Logistic regression results indicated non-significant association between being on HAART and sexual risk behaviours.

Conclusion: Contrary to anecdotal reports that availability of HAART is associated with sexual risky sexual behaviours, our results indicate non-significant statistical association between being on HAART and risky sexual behaviours. Current HAART education programs in Botswana have to be maintained to avoid risky sexual behaviours associated with HAART availability as in other contexts.
\end{abstract}

Keywords: Botswana; adults; HAART; anti-retroviral treatment; sexual behaviour.

DOI: https://dx.doi.org/10.4314/ahs.v18i3.6

Cite as: Keetile M, Kgosidintsi G. Sexual behaviours of HIV positive adults receiving HAART in Botswana: a cross sectional study. Afri Health Sci. 2018;18(3): 503-511. https:/ / dx.doi.org/10.4314/ahs.v18i3.6

\section{Introduction}

Botswana is one of the countries in sub-Saharan Africa which provide free highly active antiretroviral treatment (HAART) to HIV / AIDS infected persons. It is estimated that nationally, $90 \%$ of people with HIV who are in need of HAART had access to medication in $2011^{1}$. As a result of the availability and success of HAART in dramatically reducing morbidity and mortality from HIV/AIDS,

\section{Corresponding author: \\ Mpho Keetile, \\ University of Botswana, \\ Population Studies. \\ Email: mphokeet@yahoo.com}

many HIV/AIDS infected persons are now living longer and healthier lives, and are also sexually active.Studies in Botswana have demonstrated that most patients are good at adhering to their ARVs $>90 \%$ of the time ${ }^{2}$. The rollout of HAART has enabled the disease to move into a more chronic state, and the wide application of the treatment has been shown to have decreased AIDS-related mortality among individuals ${ }^{3}$.

However, earlier studies have indicated that unprotected sex and other risky sexual behaviours are a cause for concern among people living with HIV/AIDS (PLWHA), because they risk transmitting infection to serodiscordant partners and re-infecting themselves with drug resistant strains of the virus ${ }^{4-5}$. Recent research has also found worrying concern of reduction in protective and preventive behaviours among HIV-positive persons once their 
physical condition improved in response to anti-retroviral therapy ${ }^{5-7}$. Moreover, a review of studies on sexual risk behaviours among PLWHA by Crepaz and Marks ${ }^{7}$ shows that a considerable percentage (between 10 and 60 percent, depending on the specific sex act) of seropositive individuals continue to engage in unprotected sexual behaviours that place their partners at risk for infection and place themselves at risk for contracting secondary infections (e.g., syphilis, gonorrhoea, herpes).

Although another body of literature is showing no association between HAART use and risky sexual behaviour ${ }^{7-8}$, much of the evidence comes largely from studies conducted in developed countries and among HIVpositive people, and those at a mostly higher risk of infection such as men who have sex with men, sex workers and those in unstable unions. Little is known about the sexual behaviours of people on HAART in the context of HAART availability. Available literature in Botswana is on the perceptions and HIV risk behaviours of the general population since HAART was made available in $2002^{1 \& 9}$. The aim of this paper, therefore, is to contribute to filling this research gap by using nationally representative data to assess the extent to which the wide and free availability of HAART in Botswana, a developing country, has influenced sexual behaviours of adults on HAART.

\section{Methods}

\section{Study population}

Data used in this paper was derived from the 2013 Botswana AIDS Impact Survey (BAIS IV), which is the fourth and latest of a series of nationally representative demographic surveys aimed at providing up to date information on the Botswana's HIV / AIDS pandemic. Some of the objectives of the BAIS IV included providing latest information on the national HIV prevalence and incidence estimates among the population, 18 months and above; to provide indicative trends in sexual and preventive behaviour among the population aged 10-64 years; and provide a comparison between HIV rate, behaviour, knowledge, attitude, poverty and cultural factors that are associated with the pandemic with estimates derived from previous surveys. Data collection was done using smart phone tablets instead of the conventional paper based method.

BAIS-IV employed a national two stage sample survey design. The first stage was the selection of Enumeration Areas (EAs) as Primary Sampling Units (PSUs) selected with probability proportional to measures of size (PPS), where measures of size (MOS) were the number of households in the EA as defined by the 2011 Population and Housing Census. EAs were selected with probability proportional to size. In the second stage of sampling, the households were systematically selected from a fresh list of occupied households prepared at the beginning of the survey's fieldwork (i.e. listing of households for the selected EAs) and households were drawn systematically. Estimates for response rates showed that $83.9 \%$ of persons aged 10 to 64 answered individual questions. The data also showed that $73.4 \%$ of population 6 weeks and above participated in HIV testing. The targeted sampled population (aged 10-64 years) for BAIS IV was 9,807 and from this, 8,321 individuals were successfully interviewed yielding a response rate of $83.9 \%$.

From a total of 8,321 individuals who were successfully interviewed during BAIS IV, a total sample of 1065 HIV positive men and women in ages 20-49 years, who had successfully completed BAIS IV individual questionnaire were selected using the SPSS data selection command and were considered for analysis.

\section{Outcome variables [1]}

The main outcome variable for this paper was risky sexual behaviour, which is derived from four related variables. The following variables were used to explain sexual behaviours of people on ARVs comparing their behaviours with those of HIV positive that were not yet on HAART. a) Multiple sex partners- This was derived from a question which sought to find out the number of sexual partners a respondent had in the past twelve months preceding the survey. The variable was coded as 1 partner meant one sexual partner, while more than 1 partner meant multiple sexual partners, The resultant variable was coded such that; Multiple sex partners yes $=1$ and no $=0$.

b) Condom use consistency- Condom use consistency was measured by responses to questions that sought to find out if the respondents had always used condoms with three different sexual partners. A composite variable for condom use inconsistency with past three sexual partners was then derived from the three questions, which are 
as follows: Did you always use condoms with most recent partner in past 12 months; Did you always use condoms with next most recent partner in past 12 months and Did you always use condoms with second most recent partner in past 12 months? All the 'no' responses were summed up to indicate condom use inconsistency, while all 'yes' responses were summed up to denote consistent condom use with all partners. The resultant variable was coded such that inconsistent condom use, yes $=1$ and consistent condom use, no $=0$.

c) Intergenerational sex- This was derived from the question asking the respondent whether they have had sexualintercourse with a partner who was 10 or more years older or younger. The resultant variable was intergenerational sex, yes $=1$ and no $=0$.

d) Transactional sex- This was derived from the survey question which asked respondents whether they have ever exchanged/received gift/money for sex (transactional sex). The resultant variable was coded such that yes $=1$ and no $=0$.

\section{Exposure variable}

Sexual self-efficacy- This variable is measured by 3-item scale assessing people's confidence in their ability to practice safer sex. Participants were asked if they believed that they could persuade a sex partner to use a condom and if they could persuade a sex partner not to have sex if they were not interested. Each of the items was coded as 1 if they believed that they could persuade their sex partners all the time to use a condom or not to have sex if they were not interested and 0 if they sometimes did persuade and 2 did not believe they could persuade their partners. The resultant variable was; Persuade a partner sometimes $=0$, Always persuade partner $=1$ and Never persuade partner $=2$.

\section{Covariates}

Potential confounding variables considered were age, education, marital status, religion, and place of residence.

\section{Data analysis}

The paper applies both bivariate and multivariable analysis to examine covariates of sexual behaviours of adults on HAART. Binary logistic regression was used to evaluate the effect of a selected group of independent variables on outcome variables. Statistical Package for Social Sciences (SPSS) logistic regression results are presented as adjusted odds ratios, together with their significance levels. Four net effects model[2] were used to assess the effect of independent variables on the outcome variables. The models were used as follows; Model 1: The probability of inter-generational sex among adults on HAART; Model 2: The probability of multiple sex partners among adults on HAART; Model 3: The probability of inconsistent condom use among adults on HAART \&Model 4: The probability of transactional sex among adults on HAART.

To control for cluster effects complex samples module in SPSS has been used since BAIS IV used cluster sampling technique. The data were analysed using SPSS version 22 program.

\section{Results \\ Study sample}

Table 1 shows percentage distribution of the sample population. Females constituted a large proportion $(69 \%)$ of the study population. Adults in ages $30-34$ years (22\%), $35-39$ years $(23 \%)$ and $40-44(20 \%)$ years were the most represented groups in the sample, while age-group 20-24 years $(7 \%)$ was the least represented in the sample with respondents accounting for less than 10 per cent of the sample. When looking at residence, most respondents were from rural areas $(43 \%)$ followed by urban villages $(24 \%)$ and towns (23\%). Meanwhile, never married respondents accounted for $44 \%$ of the sample followed by cohabiting couples $(34 \%)$. Forty three percent and thirty eight per cent of respondents had primary or low and junior secondary education, respectively. 
Table 1: Percentage distribution of sample population

Sex

Percentage

31.2

68.8

AGE

Female

20-24

25-29

30-34

35-39

40-44

45-49

Place of residence

Cities

Towns

Urban Villages

Rural

Marital status

Married

Never been married

Living together

Once married

Education level

Primary \& below

Junior Secondary

Senior Secondary

Tertiary/Higher

Religious affiliation

Christian

Other non-Christian

No Religion
6.5

13.3

22.1

23.1

20.2

14.9

10.3

22.5

24.4

42.7

15.4

43.6

34.4

6.7

43.2

37.7

11.1

8.1

86.5

10.3

3.2
Number(N=1065)

332

733

69

142

235

246

215

159

110

240

260

455

164

464

366

71

460

401

118

86

920

109

36
ARV use, knowledge, sexual and behavioral practices of participants

Table 2 results indicate ARV use, knowledge and sexual behaviors of the sampled population. Results indicate that of the $1065 \mathrm{HIV}$ positive adults aged 20-49 years, just under one quarter $(22.3 \%)$ reported to be on HAART. $18.5 \% \%$ and $26.7 \%$ of respondents indicated that they never persuade or sometimes persuade their partners not to have sex or to have sex with a condom, respectively. Meanwhile $1.9 \%$ of respondents were involved in transactional sex in the 12 months preceding the survey, 9.4\% had multiple sexual partners while roughly $6.2 \%$ reported that they were once involved in inter-generational sexual relationships. Slightly over a tenth $(11.9 \%)$ of respondents did not use condoms consistently with their past three partners. 
Table 2: ARV use, knowledge and sexual and behavioral and practices

\begin{tabular}{|ccc|}
\hline & Percentage & Number (N=1065) \\
Currently taking ARVs & 22.3 & 238 \\
Yes & 77.7 & 827 \\
No & & \\
Self-Efficacy & 18.5 & 197 \\
Never persuade a partner & 26.7 & 284 \\
Persuade partner sometimes & 54.8 & 584 \\
Always persuade partner & & \\
Transactional sex in past 12 months & 1.9 & 20 \\
Yes & 98.1 & 1045 \\
No & 90.6 & 965 \\
Number of sexual partners in the past $\mathbf{1 2}$ months & 100 \\
One & 9.4 & 66 \\
Two or more & & 999 \\
Yntergenerational sex & 6.2 & \\
No & 93.8 & 938 \\
Condom use consistency & & 127 \\
Yes & 88.1 & \\
No & 11.9 & \\
\hline
\end{tabular}

About $13.3 \%$ of HIV positive adults who were not on HAART compared to $8.2 \%$ of those who were on HAART reported that they had multiple sex partners. Furthermore, $9.7 \%$ of HIV positive adults in the sample had been involved in inter-generational sex compared to only $5.2 \%$ of those on HAART. Meanwhile, inconsistent condom use was significantly highest among respondents on HAART $(13.2 \%)$ than among those who are not on HAART $(10.1 \%)$. On the other hand, transactional sex in the past 12 months was slightly high among respondents who were not on HAART $(3.1 \%)$ than among those on HAART $(1.6 \%)$.

Table 3: Percentage Distribution of HAART use by sexual behaviors

\begin{tabular}{|c|c|c|c|}
\hline Not or & RT\% $(n=827)$ & OnHAART \% $(n=238)$ & Total \\
\hline \multicolumn{4}{|c|}{ Number of sexual partners in the past 12 months } \\
\hline One/None & 86.7 & 91.8 & 965 \\
\hline Two or more & 13.3 & 8.2 & 100 \\
\hline \multicolumn{4}{|l|}{ Intergenerational sex } \\
\hline Yes & 9.7 & 5.2 & 66 \\
\hline No & 90.3 & 94.8 & 999 \\
\hline \multicolumn{4}{|c|}{ Condom use consistency } \\
\hline Yes & 10.1 & 13.2 & 571 \\
\hline No & 89.9 & 86.8 & 494 \\
\hline \multicolumn{4}{|c|}{ Transactional sex in past 12 months } \\
\hline Yes & 3.1 & 1.6 & 20 \\
\hline No & 96.9 & 98.4 & 1045 \\
\hline
\end{tabular}


The influence of HAART on sexual risk behaviours of adults

\section{Intergenerational sex}

Model 1 shows logistic regression results on the likelihood that individuals on HAART were involved in inter-generational sex 12 months prior to the survey. Results in this table indicate no significant statistical association between being involved in inter-generational sex and having multi- ple sex partners, self-efficacy and transactional sex among adults on HAART even after introducing control variables in the model. Meanwhile, results show a statistically significant association between inter-generational sex and inconsistent condom use for adults on HAART. Adults on HAART and were involved in inter-generational sex were 0.5 times less likely than those not on HAART to report inconsistent condom use.

Table 4: Odds ratios (OR) for the probability of intergenerational sex, multiple sex partners, inconsistent condom use and transactional sex among adults on HAART

\section{Model $1 \quad$ Model $2 \quad$ Model $3 \quad$ Model 4}

\begin{tabular}{|c|c|c|c|c|c|}
\hline \multicolumn{6}{|c|}{ Sexual self-efficacy } \\
\hline & Never persuade partner & 0.75 & 1.12 & $0.21 *$ & 0.75 \\
\hline & Persuade sometimes & 1.25 & 4.32 & $0.61 *$ & 0.65 \\
\hline & Always persuade partner & 1.00 & 1.00 & 1.00 & 1.00 \\
\hline \multicolumn{6}{|c|}{ 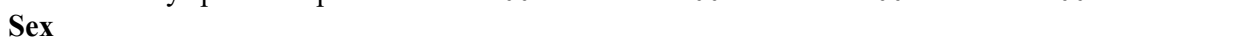 } \\
\hline & Male & 1.01 & 0.13 & 1.05 & 0.76 \\
\hline & Female & 1.00 & 1.00 & 1.00 & 1.00 \\
\hline \multirow[t]{6}{*}{ Age } & $20-24$ & 0.96 & 0.13 & 0.50 & 0.22 \\
\hline & $25-29$ & 0.87 & 0.46 & 0.89 & 0.20 \\
\hline & $30-34$ & 0.92 & 1.31 & 0.84 & 1.46 \\
\hline & $35-39$ & 0.86 & 2.01 & 0.93 & 0.53 \\
\hline & $40-44$ & 0.43 & 2.71 & 1.10 & 0.49 \\
\hline & $45-49$ & 1.000 & 1.00 & 1.00 & 1.00 \\
\hline \multicolumn{6}{|c|}{ Place of residence } \\
\hline & Cities & 0.52 & 1.58 & 1.76 & 2.23 \\
\hline & Towns & 0.77 & 0.93 & 1.39 & 1.02 \\
\hline & Urban Villages & 0.99 & 4.91 & 1.31 & 1.10 \\
\hline & Rural & 1.00 & 1.00 & 1.00 & 1.00 \\
\hline \multicolumn{6}{|c|}{ Marital Status } \\
\hline & Married & 0.59 & 5.05 & 0.81 & 0.83 \\
\hline & Never been married & 0.82 & 4.23 & 1.84 & 3.30 \\
\hline & Living together & 1.02 & 15.9 & 0.62 & 0.56 \\
\hline & Once married & 1.00 & 1.00 & 1.00 & 1.00 \\
\hline \multicolumn{6}{|c|}{ School Attendance } \\
\hline & Primary \& below & 1.20 & 4.22 & 0.62 & 2.55 \\
\hline & Junior Secondary & 1.01 & 2.85 & 0.57 & 3.99 \\
\hline & Senior Secondary & 1.12 & 1.25 & 0.39 & 0.81 \\
\hline & Tertiary/Higher & 1.00 & 1.00 & 1.00 & 1.00 \\
\hline \multicolumn{6}{|c|}{ Religious Affiliation } \\
\hline & Christian & 1.06 & 0.58 & 0.92 & 0.56 \\
\hline & Other non-Christian & 1.20 & 0.42 & 1.57 & 1.93 \\
\hline & No Religion & 1.00 & 1.00 & 1.00 & 1.00 \\
\hline \multicolumn{6}{|c|}{ Intergenerational sex } \\
\hline & Yes & 0.89 & 2.69 & 0.87 & \\
\hline & No & 1.00 & 1.00 & 1.00 & \\
\hline \multicolumn{6}{|c|}{ Number of sexual partners in the past 12 months } \\
\hline & One & 1.00 & & 1.00 & 1.00 \\
\hline Two & or more & 0.99 & & 0.28 & 2.52 \\
\hline \multicolumn{6}{|c|}{ Condom use consistency } \\
\hline & Yes & 1.00 & 1.00 & & 1.00 \\
\hline & No & 0.52 & 0.24 & & 0.52 \\
\hline \multicolumn{6}{|c|}{ Transactional sex in past 12 months } \\
\hline & Yes & 0.79 & 0.89 & 0.59 & \\
\hline & No & 1.00 & 1.00 & 1.00 & \\
\hline
\end{tabular}




\section{Multiple sex partners}

Model 2 shows logistic regression results for the probability that an individual on HAART had multiple sex partners. Results in table 4show no statistical significant association between having multiple sex partners and self-efficacy, condom use consistency, inter-generational sex and transactional sex among adults on HAART. Meanwhile, after controlling for confounders men on HAART were less likely to have multiple sex partners than those not on HAART.

\section{Inconsistent condom use}

Model 3 shows logistic regression results for the likelihood that individuals on HAART did use condoms consistently. Results indicate that individuals who were on HAART and never persuaded their partners (OR, 0.209, $\mathrm{P}=0.000$ ) or persuaded their partners sometimes (OR, $0.610, \mathrm{P}=0.026)$ to use condoms or refused sex were less likely to have used condoms consistently. Furthermore, adults who had senior secondary school education and were on ART were less likely to use condoms consistently than those with tertiary education.

\section{Transactional sex}

Model 4 shows logistic regression results for the probability that individuals on HAART were involved in transactional sex in the past 12 months prior to the survey. Results indicate no significant association between transactional sex and self-efficacy, condom use consistency, multiple sex partners and inter-generational sex. Even after introducing control variables in the model no significant association was found between transactional sex and other sexual behaviors among people on HAART.

\section{Discussion}

Some concerns have been raised that improved access to HAART could increase HIV risk behaviours creating new threats of resistance to existing anti-retroviral therapies and increasing HIV incidence ${ }^{10-12}$. The current studyhas shown that about $2 \%$ of respondents were involved in transactional sex in the 12 months preceding the survey. Although this constitutes a relatively small proportion of respondents, it raises some concerns of propelling HIV transmission. Transactional sex has been more associated with economic dependence of women on men. Evi- dence of existence of transactional sex among adults on HAART may serve to propagate further the spread of HIV/AIDS in the general population.

Inter-generational sexual relations have been thought to play an important role in the propagation of HIV in Africa. Roughly $6 \%$ of adults receiving HAART reported that they were once involved in inter-generational sexual relationships. This facilitates the intergeneration transmission of the HIV making it difficult for the government to control the spread of the virus. Inter-generational relationships have been noted to confer additional risk for HIV since young sexually active men and women indulge in sexual relationships with older men and women ${ }^{13}$.

About $9 \%$ of respondents were involved in multiple sexual partners' relationships.There are concerns that concurrency among individuals on HAART may have adverse public health effects ${ }^{14}$. Concurrent partnerships have negative implications for HIV prevention, treatment, and care efforts through the transmission of HIV to sero-negative partners or through HIV re-infection between sero-positive partners. The population health significance of these reported multiple sex relationships has been underscored by the fact that almost one in five of those on HAART who engaged in concurrency also reported inconsistent condom use $\mathrm{e}^{14}$. The results of the study underscores the importance of further investigating multiple sex partnerships among individuals on HAART and in the general population to gain a better understanding of the social and individual factors which shape this behaviour.

There have been worries in that providing HAART may lead to an increase in sexual risk behaviours such as inconsistent condom use because of the improved quality of life associated with HAART ${ }^{15-16}$. Available evidence from South Africa, Ethiopia, and Uganda, and Kenya revealed that risky sexual behaviours declined significantly after HAART initiation. A higher proportion of HIV-infected persons on HAART have reported consistent condoms use with regular partners compared with those not receiving HAART ${ }^{15,17,18}$. At multivariable level, results of the current study indicate non-significant statistical association between inconsistent condom use and being on HAART. It should be noted that even though some stud- 
ies reported reductions in risky sexual behaviour such as inconsistent condom use among HIV-infected persons, none have reported a hundred percent consistent condom use among their participants. This may suggest possibility of unprotected sex among HIV-infected persons on HAART.

\section{Limitations}

The findings of this study provide vital insights about the association between HAART availability and risky sexual behaviours in Botswana, however there are some limitations. The main limitation of the study is the use of secondary data which limited us to the variables within dataset. There is need for further qualitative investigation on how people on HAART view their sexual behaviours. The second limitation is that since data is derived from a cross-sectional survey, it limits this analysis because data on each participant are recorded only once hence it would be difficult to infer the temporal association between a risk factor and an outcome.

\section{Conclusion}

Results of this study indicate no evidence of risky sexual behaviours among HIV positive adults receiving HAART in Botswana. The findings of this study are contrary to anecdotal beliefs that HIV positive people on HAART indulge in risky sexual behaviours. There is need to maintain existing HAART education programs to avoid risky sexual behaviours associated with HAART availability in other contexts.

\section{Acknowledgement}

We wish to thank Statistics Botswana for providing us with the Botswana AIDS Impact Survey IV data set. We also wish to thank the University of Botswana, Department of Population Studies, for allowing us the time, facilities and resources to conduct this analysis.

\section{Conflict of interest}

No conflict of interest.

\section{References}

1. Gill W, Mokomane Z, Drage M. Availability of HAART and Risky Sexual Behaviour:Insights from Botswana.2008.http://iussp.org/sites/default/files/event_ call_for_papers/Extended\%20Abstract_3.pdf
2. Bussmann H. et al. Five-year outcomes of initial patients treated in Botswana's national anti-retroviral treatment program. AIDS, 2008, 22(17):2303 PubMed -2311. 3. World Health Organization. World Health Statistics.2006:WHO Press.Geneva

4. Chen SY. et al."Continuing increases in sexual risk behaviour and sexually transmitted diseases among men who have sex with men," American Journal of Public Health. 2002, 92:1387-1388.

5. Katz MH et al. 'Impact of highly active antiretroviral therapy on HIV sero-incidence among men who have sex with men: San Francisco," American Journal of Public Health. 2002; 92: 388-394.

6 . Van der Ven, P. et al. "Increasing proportions of Australian gay and homosexually active men engage in unprotected anal intercourse with regular and casual partners," AIDS Care. 2002, 14(3): 335-341.

7. Crepaz N, G Marks. "Towards an understanding of sexual risk behaviour in people living with HIV: A review of social, psychological and medical findings," AIDS 2002, 16: 135-149.

8. Elford J, Bolding G, Sherr L. High risk sexual behaviour increases among London gay men between 1998-2001: What is the role of HIV optimism? 2002.19,1537-1544

9. Keetile M. High-risk behaviors among adult men and women in Botswana: Implications for HIV/AIDS prevention efforts, SAHARA-J: Journal of Social Aspects of HIV/AIDS: An Open Access Journal, 2014,11:1, 158166, DOI: 10.1080/17290376.2014.960948

10. Moattia JP, Prudhommeb J, Traorec DC, Anne Juillet-Amaric, AJ Hortense Aka-Dago Akribic, HA Msellatid. Pand the Côte d'Ivoire Drug Access Initiative Socio-Behavioural Evaluation Group* Access to ARVT and Sexual Behaviors of HIV-Infected patients aware of their serostatus in Cote D' Ivore. AIDS 2003, 17 (suppl 3):S69-S77

11. Michael Stein, Debra S. Herman, Elizabeth Trisvan, Paul Pirraglia, Patricia Engler, Bradley J. Anderson. Alcohol Use and Sexual Risk BehaviorAmong Human Immunodeficiency Virus-Positive Persons. Alcoholism: Clinical and Experimental Research. Volume 29, Issue 5, pages 837843, May 20057.

12. Luchters S, Sarna AS, Geibel S, Cheriseh M, Munyao P, Kaai S, Mnadaliya K, Rutenberg N, Jimmerman M. Sexual risk behaviours of HIV-positive persons receiving ART in Mombasa, Kenya: Longitudinal study findings" Horizons Research Summary 2007, 1-3.

African Health Sciences Vol 18 Issue 3, September, 2018 
13. Leclerc-Madlala S. Age-Disparate and Intergenerational Sex in Southern Africa: The Dynamics of Hyper Vulnerability. AIDS, 2008, 22(Suppl. 4), S17-S25. doi: 10.1097/01.aids.0000341774.86500.53

14. Brendan Maughan-Brown Atheendar S. Venkataramani Timothy L. Mah Concurrent sexual partnerships among individuals on HAART in South Africa: An opportunity for HIV prevention, 2009. CSSR Working Paper No. 265, South Africa.

15. Sarna A, Luchters S, Geibel S, Kaai S, Munyao P, et al. Sexual Risk Behaviour and HAART: A Comparative Study of HIV-Infected Persons on HAART and on Preventive Therapy in Kenya. International Journal of STD \& AIDS, 2008, 19, 85-89 PubMed . http://dx.doi. org/10.1258/ijsa.2007.007097

16. Eisele TP, Mathews C, Chopra M, Lurie MN, Brown $\mathrm{L}$ et al. Changes in Risk Behavior among HIV-Positive Patients during Their First Year of Antiretroviral Therapy in Cape Town South Africa. AIDS and Behavior, 2009, 13, 1097-1105 PubMed . http://dx.doi.org/10.1007/ s10461-008-9473-2
17. Wandera B, Kamya MR, Castelnuovo B, Kiragga A, Kambugu A, et al. Sexual Behaviors over a 3-Year Period among Individuals with Advanced HIV/AIDS Receiving Antiretroviral Therapy in an Urban HIV Clinic in Kampala, Uganda. Journal of Acquired Immune Deficiency Syndromes, 1999, 57, 62-68. http://dx.doi.org/10.1097/ QAI.0b013e318211b3f2

18. Venkatesh KK, de Bruyn G, Lurie MN, Mohapi L, Pronyk P et al. Decreased Sexual Risk Behavior in the Era of HAART among HIV-Infected Urban and Rural South Africans Attending Primary Care Clinics. AIDS (London, England), 2010, 24, 2687-2696. http://dx.doi. org/10.1097/QAD.0b013e32833e78d4

[1]Some outcome variables were also used as covariates for models which they were not used as outcomes.

[2] Gross effects models were used to assess the relationship between sexual risk behaviours and being on HAART. These models are not presented in this article because they also did not yield any significant association with the outcome variable. 